Draft VERsion OCtOBER 26, 2018

Preprint typeset using IATEX style emulateapj v. 03/07/07

\title{
DIRECT OBSERVATION OF HIGH-SPEED PLASMA OUTFLOWS PRODUCED BY MAGNETIC RECONNECTION IN SOLAR IMPULSIVE EVENTS
}

\author{
TONGJIANG WANG ${ }^{1}$, LINHUI SUI ${ }^{2,3}$, JIONG QIU ${ }^{1}$ \\ Received 2007 February 22; ACCePted 2007 April 20; Published 2007 May $15^{4}$ \\ Draft version October 26, 2018
}

\begin{abstract}
Spectroscopic observations of a solar limb flare recorded by SUMER on SOHO reveal, for the first time, hot fast magnetic reconnection outflows in the corona. As the reconnection site rises across the SUMER spectrometer slit, significant blue- and red-shift signatures are observed in sequence in the Fe XIX line, reflecting upflows and downflows of hot plasma jets, respectively. With the projection effect corrected, the measured outflow speed is between $\sim 900-3500 \mathrm{~km} \mathrm{~s}^{-1}$, consistent with theoretical predictions of the Alfvénic outflows in magnetic reconnection region in solar impulsive events. Based on theoretic models, the magnetic field strength near the reconnection region is estimated to be $19-37$ Gauss.

Subject headings: Sun: corona — Sun: flares — Sun: UV radiation — Sun: X-rays, gamma rays
\end{abstract}

\section{INTRODUCTION}

It is generally accepted that the free magnetic energy stored in the coronal magnetic field is explosively released through magnetic reconnection Priest \& Forbes 2000), a process in which oppositely-directed magnetic field lines break and reconnect with each other in a small area in the corona. The residual magnetic tension in the newly-reconnected field causes the magnetic field and plasma to be expelled from both sides of the reconnection region, forming high-speed outflows at near-Alfvén speed ( $\sim$ thousands of $\mathrm{km} \mathrm{s}^{-1}$ ). Indirect observational evidence have been found in past studies, e.g., the separation motion of flare ribbons formed in the lower atmosphere, cusp-shaped soft X-ray flare loops (Tsuneta et al. 1992), loop-top hard X-ray sources (Masuda et al. 1994), and double hard X-ray sources above the flare loop top with opposite temperature gradients (Sui \& Holman 2003).

The search for inflows and outflows is important since they are direct consequence of the reconnection process and their measurements can yield estimates of the reconnection rate. There have been some observations interpreted as the inflow or outflow. Apparent bidirectional motion of coronal structures around a flare loop discovered with SOHO/EIT has been considered to be the signature of reconnection inflow (Yokoyama et al. 2001). There has been, however, only indirect evidence of reconnection outflows, mostly based on morphological changes. For example, plasma blob ejections (Shibata et al. 1995) and downward plasma motions (McKenzie \& Hudson 1999) seen in X-ray images were thought to be a consequence of upward or downward outflows, but the deduced outflow velocities are much lower than predicted values. In this paper, we present the first direct measurement of high-speed reconnection outflows in a flare event using combined imaging and

\footnotetext{
${ }^{1}$ Department of Physics, Montana State University, Bozeman, MT; wangtj@solar.physics.montana.edu

2 Department of Physics, Catholic University of America, 620 Michigan Avenue, Washington, DC

${ }^{3}$ Heliophysics Science Division, Code 671, NASA Goddard Space Flight Center, Greenbelt, MD

${ }^{4}$ Published in ApJ, 661, L207-L210, 2007
}
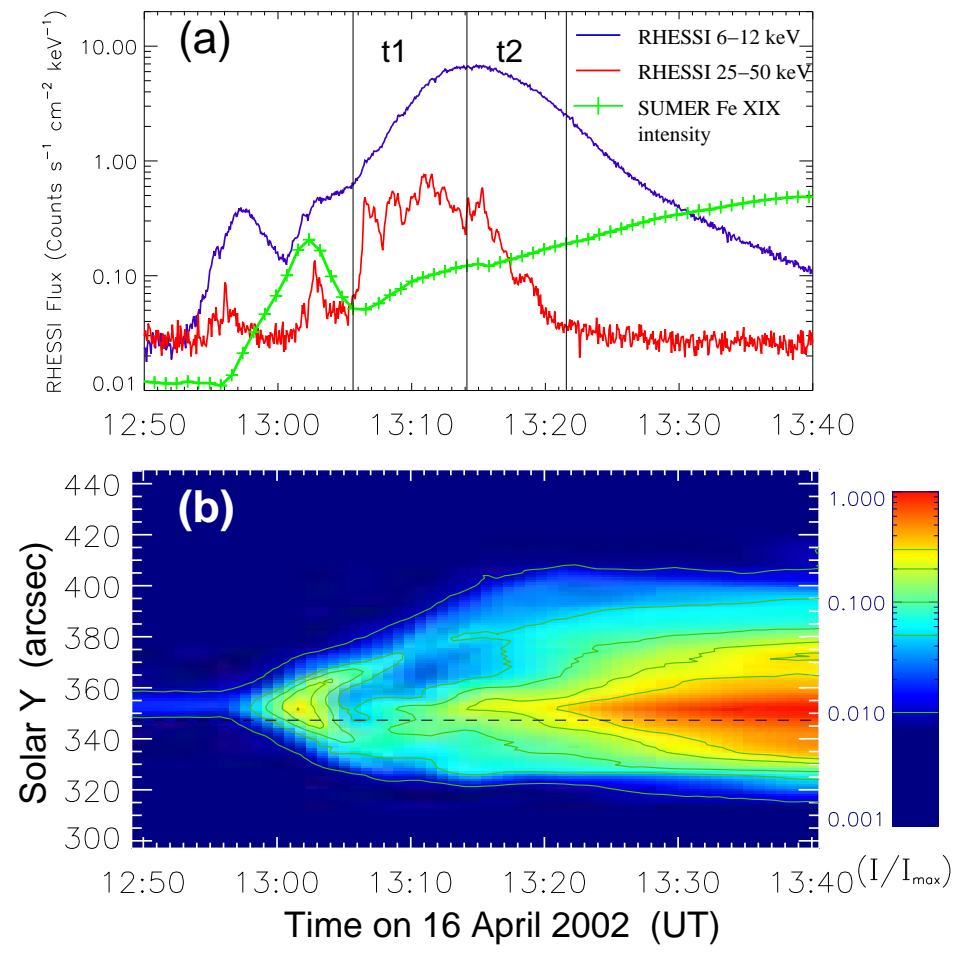

FIG. 1.- Flux evolution of an M2.5 flare on April 16, 2002, obtained with RHESSI and SUMER. (a) RHESSI light curves at 6-12 (blue curve) and 25-50 keV (red curve) energy bands. The overlaid green curve is the time profile of the Fe XIX line intensity at the slit position (dashed line in panel $(b)$ ) where a high blueshift jet (see Fig. $3 b$ ) was located. The time range marked with $t 1$ is when the high blue-shift jet was seen (top two rows of Fig. 4); the time range marked $t 2$ is when the high red-shift jet was seen (bottom row of Fig.4). (b) Time series of the Fe XIX line intensity (on a logarithmic scale) at a fixed slit position observed by SUMER. The overlaid contours indicate $1,5,10,20$, and $30 \%$ of the peak intensity in the image.

spectroscopic observations.

\section{OBSERVATIONS}

The flare studied in the paper occurred very close to the northwest limb of the Sun in the active region NOAA 9901 on 16 April 2002. It was a GOES M2.5-class 

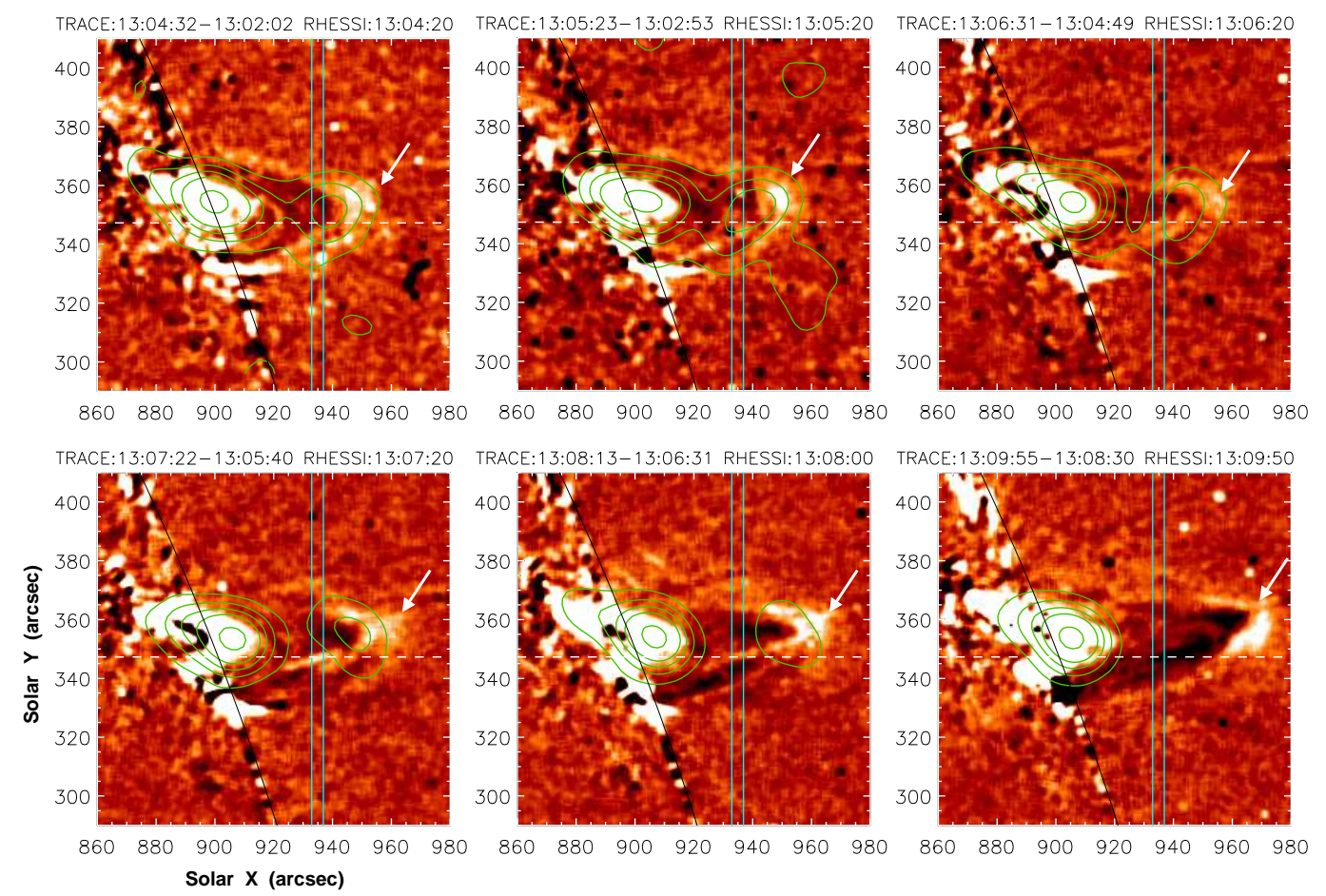

FIG. 2.- RHESSI 6-12 keV images overlaid on the TRACE $195 \AA$ difference images during the impulsive phase of the flare. Evolution of a rising faint coronal loop (marked by an arrow) can been seen in TRACE with the dark regions representing the loop seen in the earlier map and the white regions representing the loop in the current map. The RHESSI contour levels are 15, 30, 45, 60, and 90\% of the peak flux in each image. The RHESSI images were reconstructed with the CLEAN algorithm using grid 3-9, giving a resolution about $7^{\prime \prime}$. The location of the SUMER slit is indicated by the two vertical, solid lines. The horizontal dashed line indicates the position of the high blue-shift jet. An animation of this figure is available online (http://solar.physics.montana.edu/wangtj/outflow_mov.gif).

flare and associated with a slow coronal mass ejection (Goff et al. 2005). The X-ray light curves in two energy bands (6-12 and 25-50 keV) obtained with the Ramaty High-Energy Solar Spectroscopic Imager (RHESSI) are shown in Figure 1 $a$. The flare started with enhancement in soft X-rays at 12:52 UT. The impulsive phase as shown in $>25 \mathrm{keV}$ hard X-ray (HXR) started at 13:06 UT and lasted $\sim 11 \mathrm{~min}$. The RHESSI X-ray images below 25 keV show a compact flare loop and a separated, outward moving coronal source (Fig. 2, contour images). Figure2 also shows the $195 \AA$ bandpass images obtained with the Transition Region and Coronal Explorer (TRACE), in which a bright, compact loop and a faint, outward moving loop can be identified. Goff et al. (2005) reported that the faint loop was rising with a velocity of $45-75$ $\mathrm{km} \mathrm{s}^{-1}$. The outward moving X-ray coronal source appeared to be trailing the front of the large-scale loop seen in TRACE images (indicated by an arrow in Fig. (2).

The flare was observed throughout its duration by the Solar Ultraviolet Measurements of Emitted Radiation (SUMER) spectrometer on SOHO with the $300^{\prime \prime} \times 4^{\prime \prime}$ entrance slit at a fixed position above the active region (Figs. 2 and 3a). The spectra were recorded with a $50 \mathrm{~s}$ cadence in three lines: Si III 1113.2 $\AA$ (0.06 MK), Ca X $557.7 \AA$ (2nd order, 0.6 MK), and Fe XIX 1118.1 $\AA$ (8 $\mathrm{MK})$. Figure $1 b$ shows the time series of the Fe XIX line intensity profiles along the slit. From 12:58 to 13:04 UT, the line intensity increased significantly, appearing as a crescent-shaped structure passing the slit. The timing and location (relative to the slit) of this structure suggest that it is the moving, large-scale (faint) loop seen by TRACE (see Fig. 2). This structure is not seen in the
SUMER cooler Ca X line (Fig. 3b), indicating that the loop seen at TRACE 195 bandpass was due to emissions of hotter lines (i.e., Ca XVII $193 \AA$ at $5 \mathrm{MK}$ and Fe XXIV $192 \AA$ at $20 \mathrm{MK}$ ) (Warren et al. 1999). After the passage of this rising loop, the Fe XIX intensity decreased by about an order of magnitude (from 13:04 to 13:08 UT). Then it gradually increased again over a larger region, seen as a cusp-shaped brightening (Fig. 1b), which indicates apparently-growing hot, dense cusp-shaped loops across the slit. This could be the evidence of progressive reconnection of field lines towards higher altitudes in time.

The most striking signature in SUMER observations is a plasma jet with a large blue-shift in the Fe XIX line (Figs. $3 b$ and 4 ) lasting for $\sim 8 \mathrm{~min}$ during the impulsive phase of the flare. An animation for simultaneous TRACE and SUMER observations is available online ${ }^{5}$. The jet had a width of $\sim 6^{\prime \prime}$ along the slit, and was located at $\sim 5^{\prime \prime}$ south of the top of the faint, rising loop. This high blue-shift jet was first seen at 13:05 UT, when the main impulsive phase of the flare started as revealed by the RHESSI 25-50 keV light curve (Fig. 1 a). At this time, both the top of the erupting hot loop seen by SUMER and TRACE and the X-ray coronal source seen by RHESSI had passed the slit. Therefore, the highspeed outflow was trailing the coronal features moving outwards. The Fe XIX line profile shows blue-shift component corresponding to a line-of-sight Doppler velocity of up to $600 \mathrm{~km} \mathrm{~s}^{-1}$ (Fig. 3 $\mathrm{c}$ ). Taking into account the projection effect, the outflow velocity can be as high as $1800-3500 \mathrm{~km} \mathrm{~s}^{-1}$ (see discussions in Sect 3). Figure 4

\footnotetext{
${ }^{5}$ in http://solar.physics.montana.edu/wangtj/outflow_mov.gif
} 

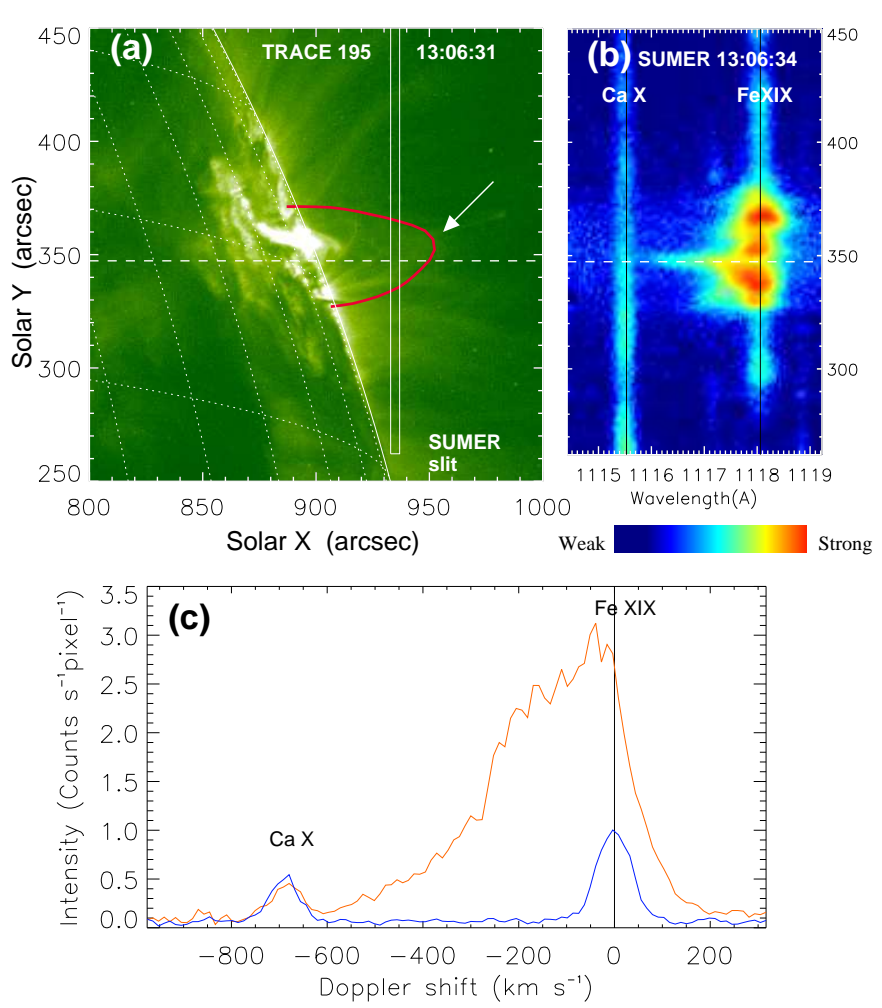

FIG. 3.- Observations of a high-speed plasma outflow. (a) TRACE $195 \AA$ image showing the compact flare loop. A sketch of the faint, rising loop (red curve) as seen in the TRACE difference image (Fig. 2) is also indicated to show its spatial relation to the SUMER slit. The SUMER slit position is co-aligned with the TRACE image with an accuracy of $\sim 1^{\prime \prime}$ in the Y-direction based on common features seen in both the SUMER Ca X line and TRACE intensity profiles along the slit. (b) SUMER spectra along the slit in a window containing a coronal line, Ca X $557.7 \AA$ (2nd order) and a hot flare line, Fe XIX $1118.1 \AA$. (c) The spectral line profile of Fe XIX (red curve) at the highly blue-shifted position (marked by the dashed line in $(b)$ ), indicating a plasma flow with the line-ofsight component up to $600 \mathrm{~km} \mathrm{~s}^{-1}$. The blue curve is the Fe XIX line profile taken about a half hour before the event, showing the stationary profile.

shows the jet evolution as observed in Fe XIX spectra. We do not see any significant change in the line profile during the first $4 \mathrm{~min}$, although the line intensity continued to increase (Fig. 11a, green line). The jet became weaker after 13:10 UT, and eventually disappeared at 13:14 UT.

A high red-shift jet in the Fe XIX line, although not as strong as the blue-shift jet, is also observed after 13:16 UT (Fig. 4, bottom row). This indicates a downward-moving plasma flow passing through the slit. The red-shift jet was at $\sim 5^{\prime \prime}$ north of the blue-shift jet. The maximum Doppler velocity was $300 \mathrm{~km} \mathrm{~s}^{-1}$. With the projection effect corrected, we get a downward velocity between 900 and $1800 \mathrm{~km} \mathrm{~s}^{-1}$. This red-shift jet disappeared after 13:21 UT when the 25-50 keV HXR emission dropped to the background level (Fig. 11a).

\section{DISCUSSIONS}

We note that, since the blue-shift jet seen by SUMER was located near the southern leg of the rising faint loop seen by TRACE (Fig. 2. panels 3-5), it may be falsely interpreted as the signature of upward flows in the loop. However, there are several arguments against such an interpretation: (1) As both the northern and southern legs of the rising loop crossed the slit, if the blue shift is caused by the upward flow in the loop, then we would expect to see two strong Doppler-shift jets along the slit; (2) After the southern leg of the TRACE loop passed through the jet location, we still observed the high blueshift jet (Fig. 2] bottom right panel). (3) A plasma flow with such a high speed is not expected and has never been reported in erupting loops.

By combining all the observations presented above, we conclude that the observed upward and downward jets are both high-speed outflows from the magnetic reconnection site. When the upflowing jet was observed, the reconnection site was below the slit and later when the downflowing jet was observed, the reconnection site had moved above the slit. We interpret the essential observations in terms of the standard magnetic reconnection picture (see Fig. 6): (1) The initial faint, rising loop observed with SUMER and TRACE is an erupting (twisted) flux rope, below which magnetic reconnection took place. The high temperature $(>6 \mathrm{MK})$ of this loop suggests the presence of a pre-heating process which may be involved in the trigger of its eruption. (2) At the onset of the flare impulsive phase, the high-speed upflows were expelled outward from the magnetic reconnection region. This plasma upflow passed through the SUMER slit and produced the high blue-shift jet seen in the Fe XIX line. The estimated upflow speed agrees with the typical Alfvén speed in the corona. (3) After the reconnection site (e.g., current sheet) passed through the slit, the observation showed the cusp-shaped, newlyreconnected field retracting downwards and forming reconnection downflows, which were observed as the redshift jet by SUMER. The relatively smaller downflow velocity may imply that the downflow is slowed down by the density increase due to chromospheric evaporation along the reconnected loops. Similar asymmetry between upflow and downflow velocities was also found in bi-directional jets observed in explosive events in the solar chromosphere (Innes et al. 1997). (4) After the impulsive phase, the reconnection rate decreased significantly (inferred from its temporal correlation with hard X-ray flux, Qiu et al. 2004) and, therefore, no high-speed reconnection outflow can be detected with SUMER. (5) The narrow width of the observed outflows suggests the presence of a vertical current sheet in side view. (6) The X-ray coronal source is closer to the reconnection region than the rising loop, therefore, it has a higher temperature (30 MK) (Sui 2004). Both this above-the-loop X-ray coronal source and the lower compact X-ray source could be heated by termination shocks formed by the reconnection outflows above and below the current sheet (Tsuneta 1997; Masuda et al. 1994). These interpretations are consistent with the results of Sui \& Holman (2003); Sui. Holman, \& Dennis (2004); Sui (2004). Based on the RHESSI observations that the temperature of the flare loop increased toward higher altitude and the temperature of the above-the-loop coronal source increased toward lower altitude in the event studied here and another homologous event, Sui et al. concluded that the magnetic reconnection occurred between the flare loop and the above-the-loop coronal source.

Now we estimate the true jet speed based on Figure 5 If we assume the outflow is radial from the active re- 

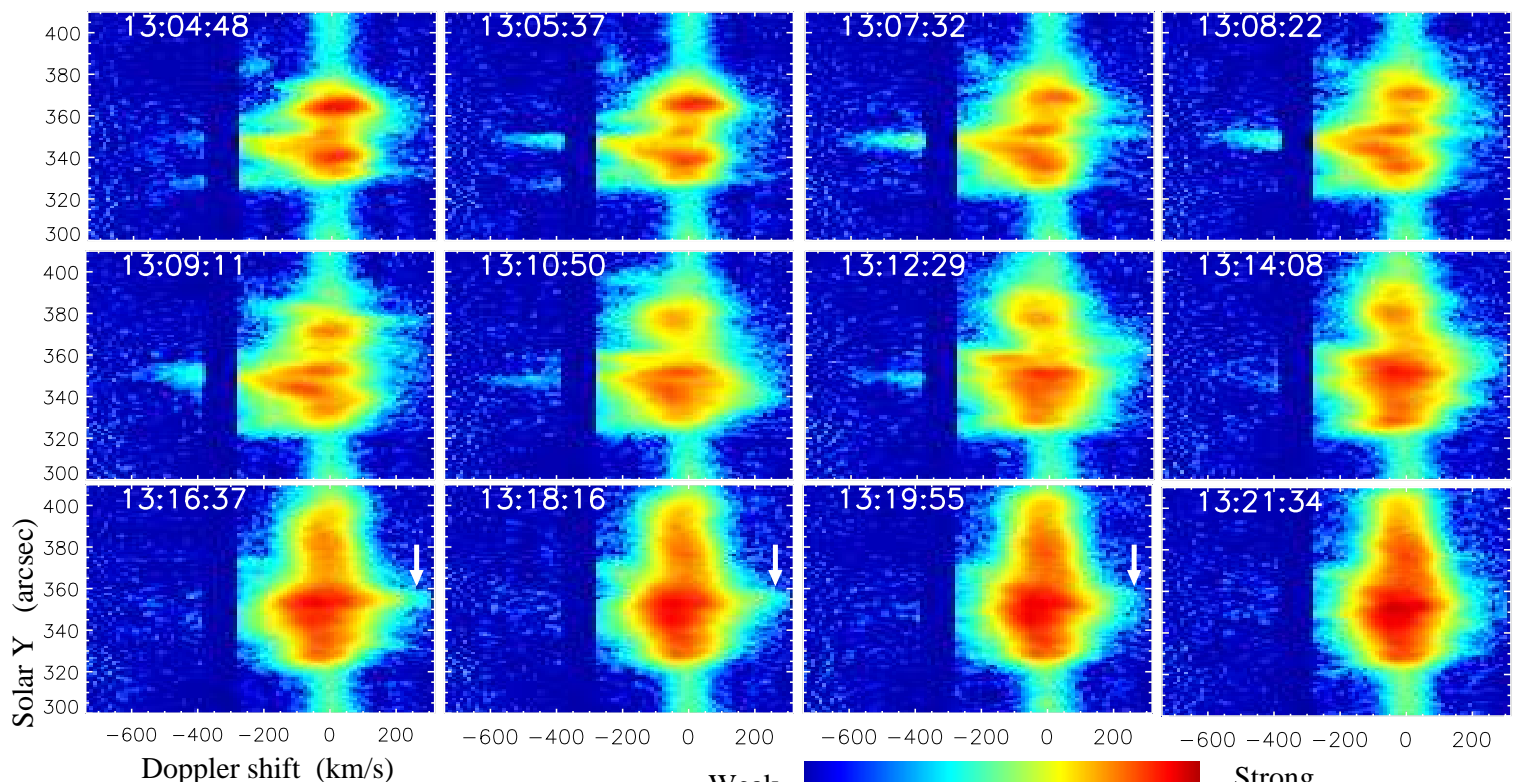

Doppler shift $(\mathrm{km} / \mathrm{s})$

\section{Weak}

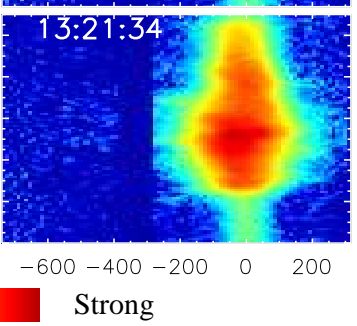

FIG. 4.- Time series of SUMER Fe XIX spectra showing a high blue-shift jet (top two rows) and a red-shift jet (marked by an arrow in bottom row), indicating a high-speed plasma upflow and downflow, respectively. The images are on a logarithmic scale. The continuum background and $\mathrm{Ca} \mathrm{X}$ line emissions have been subtracted. There is a data gap between -395 and $-276 \mathrm{~km} \mathrm{~s}^{-1}$ in each image. An animation of this figure is available online (http://solar.physics.montana.edu/wangtj/outflow_mov.gif).

gion, then it would be at $\sim 80^{\circ}$ relative to the line-ofsight. If we fit the inner side of the TRACE compact flare loops by a circular arc in a 3-dimensional geometry (Aschwanden et al. 2002) and assume that the outflow is in the direction of the line linking the midpoint of the two loop feet and the loop apex, the angle is $\sim 70^{\circ}$. Thus, the maximum blue shift of $600 \mathrm{~km} \mathrm{~s}^{-1}$ gives an outflow velocity as high as $1800-3500 \mathrm{~km} \mathrm{~s}^{-1}$ taking into account the projection effect. Since the reconnection outflows had a velocity close to the Alfvén speed $\left[V_{A} \simeq\right.$ $3000(B / 20 \mathrm{G})\left(n_{e} / 2 \times 10^{8} \mathrm{~cm}^{-3}\right)^{-1 / 2} \mathrm{~km} \mathrm{~s}^{-1}$ ] (where $B$ is the magnetic field and $n_{e}$ is the electron density), we can estimate the magnetic field strength near the reconnecting region. Given $n_{e} \sim 5 \times 10^{8} \mathrm{~cm}^{-3}$ as the average active region coronal density at a height of $\sim 40 \mathrm{Mm}$ (SUMER slit height) (Del Zanna \& Mason 2003), with $V_{A}$ of $1800-3500 \mathrm{~km} \mathrm{~s}^{-1}$, we get $B \simeq 19-37$ Gauss, which agrees well with the mean magnetic field strength of coronal loops at similar heights measured from observations of coronal loop oscillations (Nakariakov \& Ofman 2001; Aschwanden et al. 2002; Wang, Innes, \& Qiu 2007). The measurements of outflow speed and magnetic field strength near the reconnection region are essential for our understanding of the plasma heating and particle acceleration in flares.

High temperature flows of $\sim 1000 \mathrm{~km} \mathrm{~s}^{-1}$ have been observed with SUMER in a couple of other flares (Innes et al. 2001; Innes. McKenzie, \& Wang 2003). However, since observations of those events provide no information of the location of the reconnection region, it is difficult to pinpoint the origin of these flows, or directly relate these flows to the energy release region. For example, Innes, McKenzie, \& Wang (2003) observed blue shifts of $800-1000 \mathrm{~km} \mathrm{~s}^{-1}$ in the Fe XXI line from the boundary along the tail of dark downflows. The physical nature of dark downflows is not fully understood. McKenzie \& Hudson (1999) interpreted the dark downflows as signatures of reconnection outflows, though

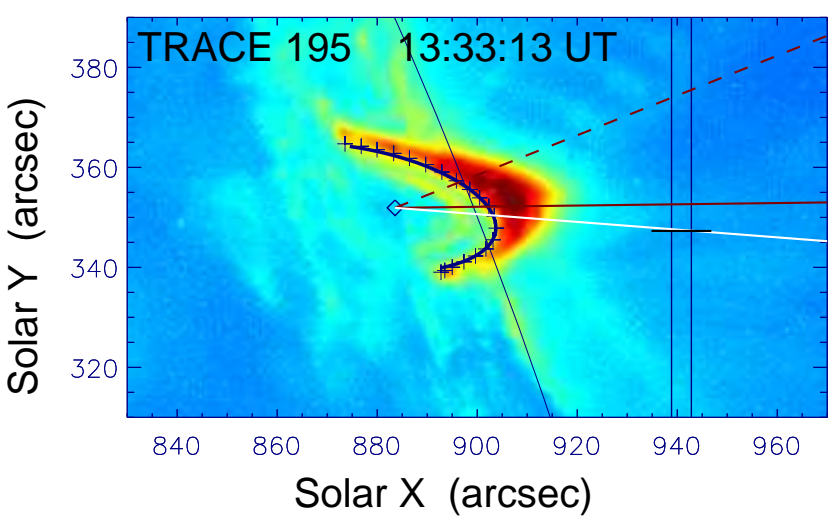

FIG. 5.- Postflare loops seen in TRACE 195 $\AA$ image at 13:33 UT. The inner side of the loop (outlined with plus signs) is fitted with a circular arc (black curve) in a 3-D geometry. The radial direction (dashed line) from the midpoint of the loop baseline has an angle to the line-of-sight (LOS) of $80^{\circ}$. The line linking the midpoint of the two footpoints to the loop apex (dark solid line) is indicated to have an orientation towards ( $\sim 5^{\prime \prime}$ above) the highshift jet seen by SUMER. The direction of this line has an angle to the LOS of $70^{\circ}$. The white line representing a direction in the loop plane and pointing just towards the jet position has an angle to the LOS of $80^{\circ}$. Therefore, we assume the outflows in the direction which has an angle to the LOS between $70^{\circ}$ and $80^{\circ}$ in the text.

the speeds are slower than typically expected and high Doppler shifts at the tail boundary are hardly explained in their picture. Innes, McKenzie, \& Wang (2003) suggested another way that these shifts may be due to a fast wind generated behind the downflows.

The combined observations of SUMER, RHESSI, and TRACE of a very well observed event studied in this paper allow us to determine unambiguously the spatial relationship between the high Doppler-shift flows and the reconnection region, and thus provide direct evidence of high-speed magnetic reconnection outflows in the current sheet in the corona. The observations lend strong support to the magnetic reconnection theory and the bipo- 


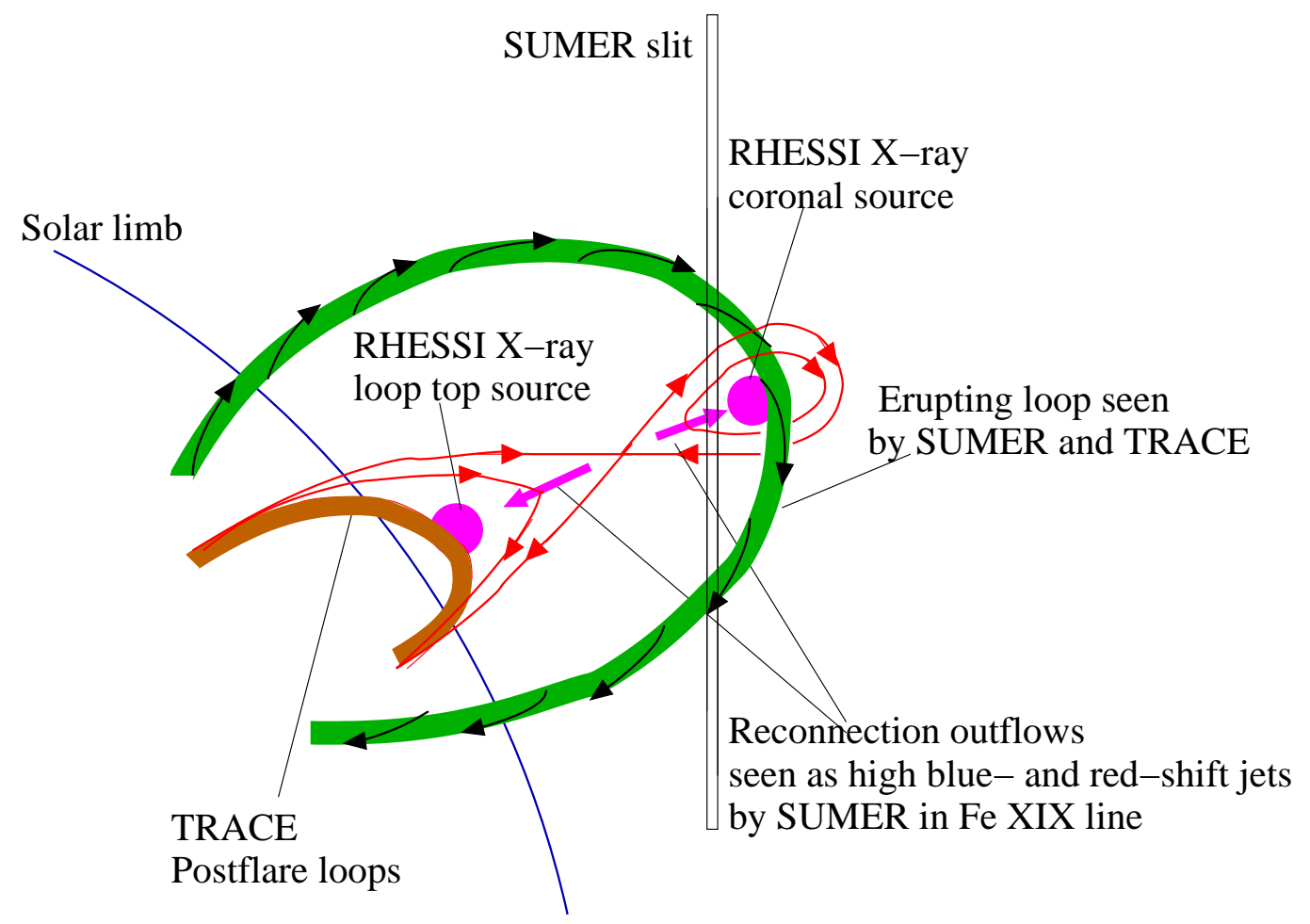

FIG. 6. - A schematic interpretation of the observed EUV and X-ray fea- tures in the flare in terms of the standard flare model

lar reconnection model of solar eruptive events. To better understand magnetic reconnection on the Sun, it requires a further analysis based on 3D models which have shown many different properties from the 2D CSHKP model (e.g., Archontis et al. 2005).

We thank D. E. Innes, B. R. Dennis and G. D. Holman for their valuable discussions and suggestions. We also thank Max-Planck-Institut für Sonnensystemforschung for providing the SUMER data. T.W. and J.Q.'s work are supported by NASA grant NNG06GA37G and NASA grant NAS5-38099. L.S.'s work is supported by NASA grant 370-16-20-16 and the RHESSI project.

\section{REFERENCES}

Archontis, V., Moreno-Insertis, F., Galsgaard, K., \& Hood, A. W. 2005, ApJ, 635, 1299

Aschwanden, M. J., et al. 2002, Sol. Phys., 206, 99

Del Zanna, G., \& Mason, H. E., A\&A, 406, 1089

Goff, C. P., et al. 2005, A\&A, 434, 761

Innes, D. E., et al. 1997, Nature, 386, 811

Innes, D. E., et al. 2001, ApJ, 549, L249

Innes, D. E., McKenzie, D. E., \& Wang, T. J. 2003, Sol. Phys., 217,267

Masuda, S., et al. 1994, Nature, 371, 495

McKenzie, D. E., \& Hudson, H. S. 1999, ApJ, 519, L93

Nakariakov, V. M., \& Ofman, L. 2001, A\&A, 372, L53

Priest, E. R., \& Forbes, T. 2000, Magnetic Reconnection - MHD Theory and Applications (Cambridge Univ. Press, Cambridge, 2000).
Qiu, J., et al., 2004, ApJ, 604, 900

Shibata, K., et al. 1995, ApJ, 451, L83

Sui, L. 2004, Ph.D. thesis (Catholic Univ. America)

Sui, L., \& Holman, G. D. 2003, ApJ, 596, L251

Sui, L., Holman, G. D., \& Dennis, B. R. 2004, ApJ, 612, 546

Tsuneta, S., et al. 1992, PASJ, 44, L63

Tsuneta, S. 1997, ApJ, 483, 507

Wang, T. J., Innes, D. E., \& Qiu, J. 2007, ApJ, 656, 598

Warren, H. P., et al. 1999, ApJ, 527, L121

Yokoyama, T., et al. 2001, ApJ, 546, L69 